\section{LAUNCHING THE ETHIOPIA HEALTH CARE QUALITY INITIATIVE: INTERIM RESULTS AND INITIAL LESSONS LEARNED}

${ }^{1}$ Hema Magge, ${ }^{2}$ Abiyou Kiflie, ${ }^{2}$ Zewdie Mulissa, ${ }^{2}$ Mehiret Abate, ${ }^{2}$ Abera Biadgo, ${ }^{2}$ Befikadu Bitewulign, ${ }^{2}$ Hareg Alemu, ${ }^{3}$ Kathryn Brooks, ${ }^{4}$ Hassen Mohammed, ${ }^{4}$ Daniel Burssa. 'Institute for Healthcare Improvement; Brigham and Women's Hospital, Ethiopia; ${ }^{2}$ Institute for Healthcare Improvement, Ethiopia; ${ }^{3}$ Institute for Healthcare Improvement, US; ${ }^{4}$ Ethiopia Federal Ministry of Health, Ethiopia

\subsection{6/bmjoq-2017-|HI.4}

Background Since 2013, IHI has worked with the Ethiopian Federal Ministry of Health $(\mathrm{FMoH})$ to leverage QI methodologies to accelerate progress of the $\mathrm{FMoH}$ in maternal newborn health $(\mathrm{MNH})$, and build a culture of quality in the health system.

Objectives Understand initial successes, challenges, and results of a multi-faceted strategy aimed at institutionalising QI and improving $\mathrm{MNH}$ outcomes in a resource-limited setting.
Methods Three collaboratives launched in three regions of Ethiopia April-September 2016. We report programmatic and core clinical indicators after LS1 (October 2016-June 2017). Monthly means of program process measures were extracted from routine monitoring tools. Control charts for core HMISderived indicators use baseline data (July 2015-August 2016) to set control limits and extend forward. Bundle implementation is displayed using run charts.

Results Currently, LS3 has been completed, and 57 health care workers have been trained in QI along with 83 manager-level staff. 20 QI teams have conducted 506 PDSA cycles. Sixteen $(80 \%)$ teams have reported signals of improvement in bundle adherence by LS2. Aggregate control charts demonstrate initial improvement in data quality in 4-visit ANC, PNC within 48 hours of delivery, and skilled delivery as seen by an initial apparent performance drop. ANC coverage, PNC coverage, percent of women tested for syphilis during first ANC visit, and clinical bundles continued with signals of improved care quality with upward trend (see charts).

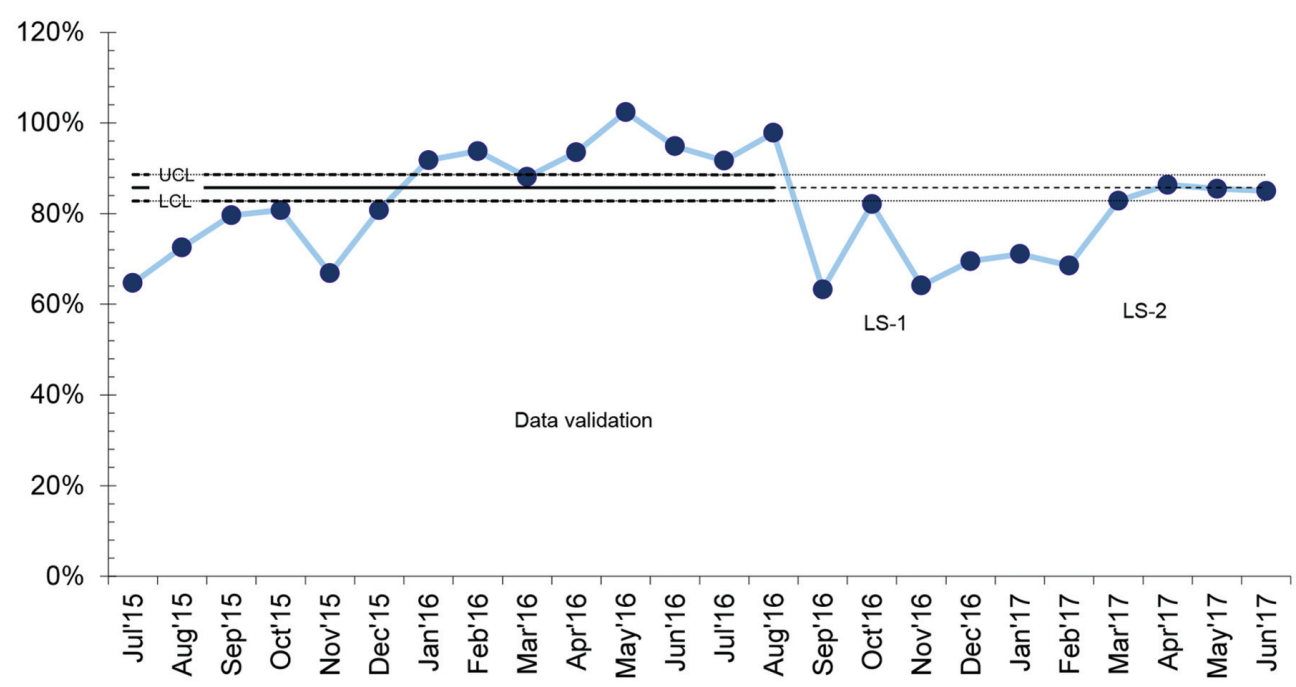

Abstract 865 Figure 14 visit ANC

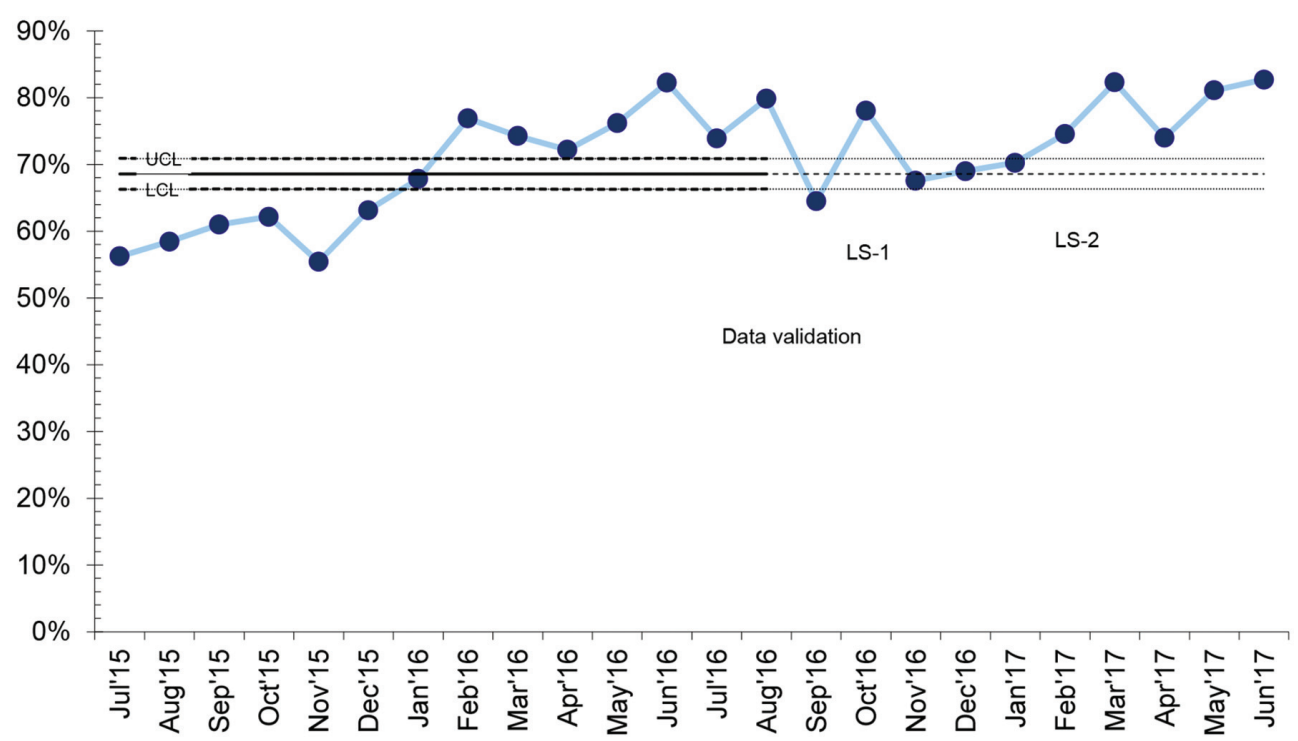

Abstract 865 Figure 2 Postnatal care within 48 hours 


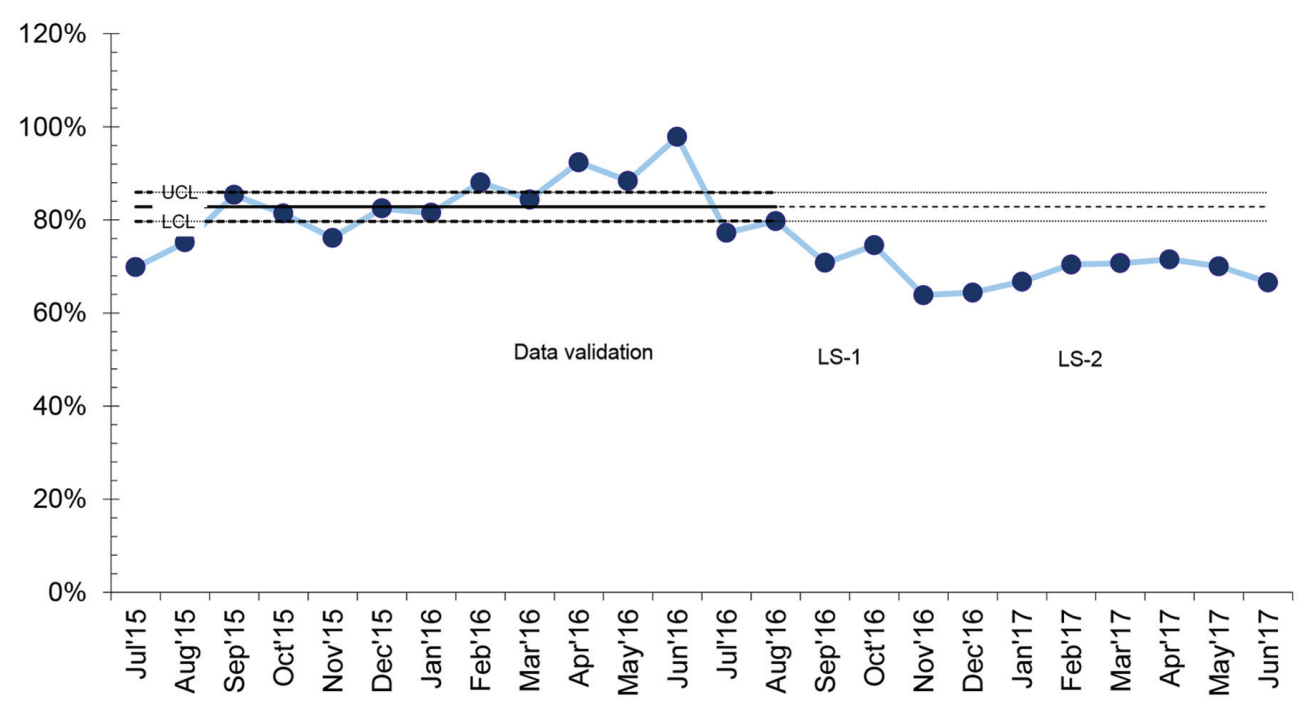

Abstract 865 Figure 3 Skilled birth attendance

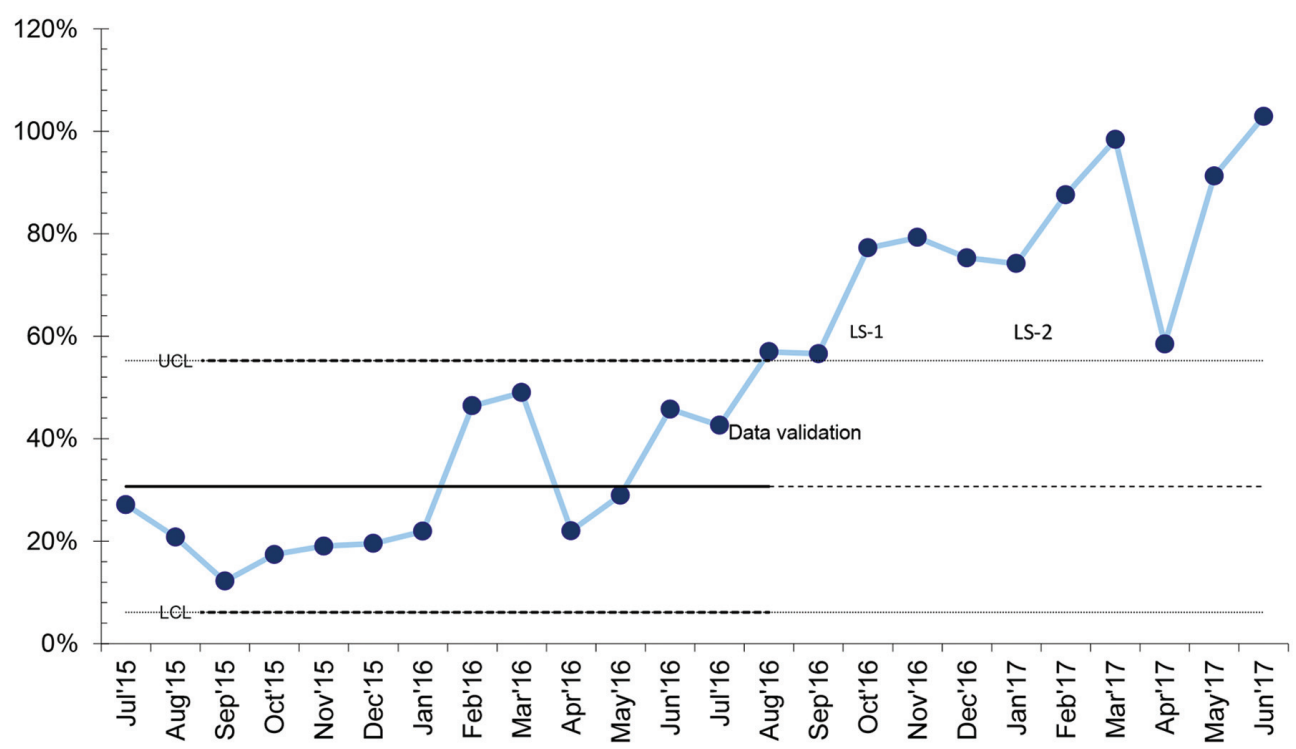

Abstract 865 Figure 4 Syphilis testing in ANC

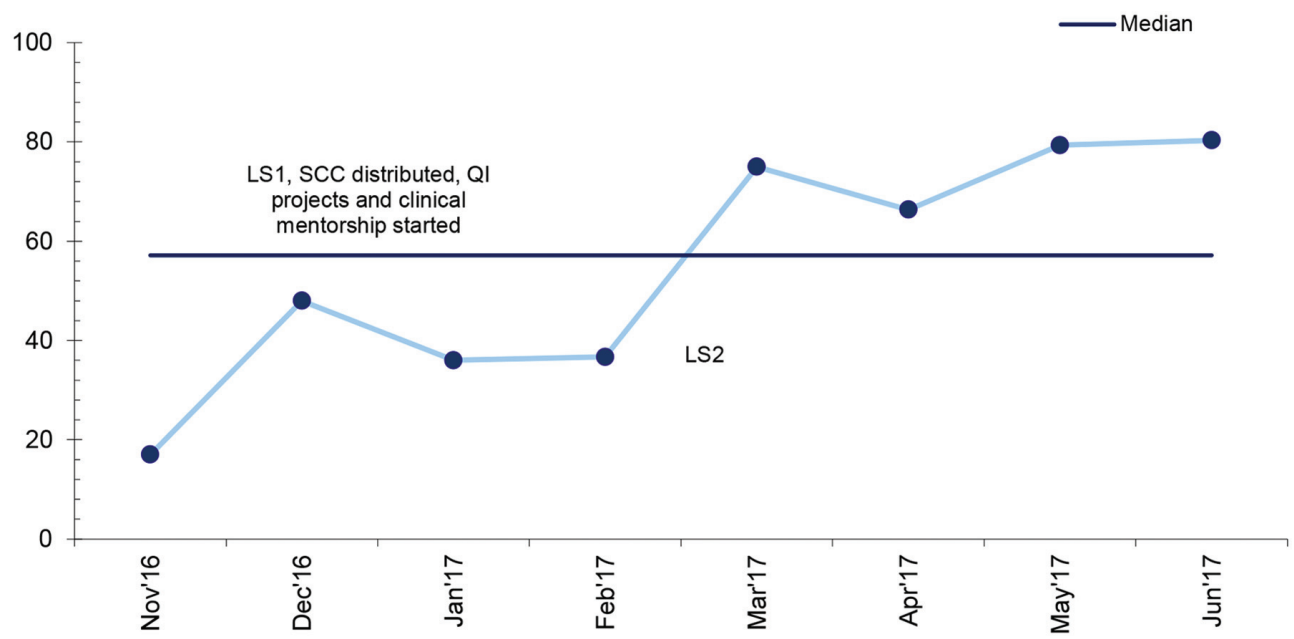

Abstract 865 Figure $5 \quad 100 \%$ completion of 'on admission' bundle 


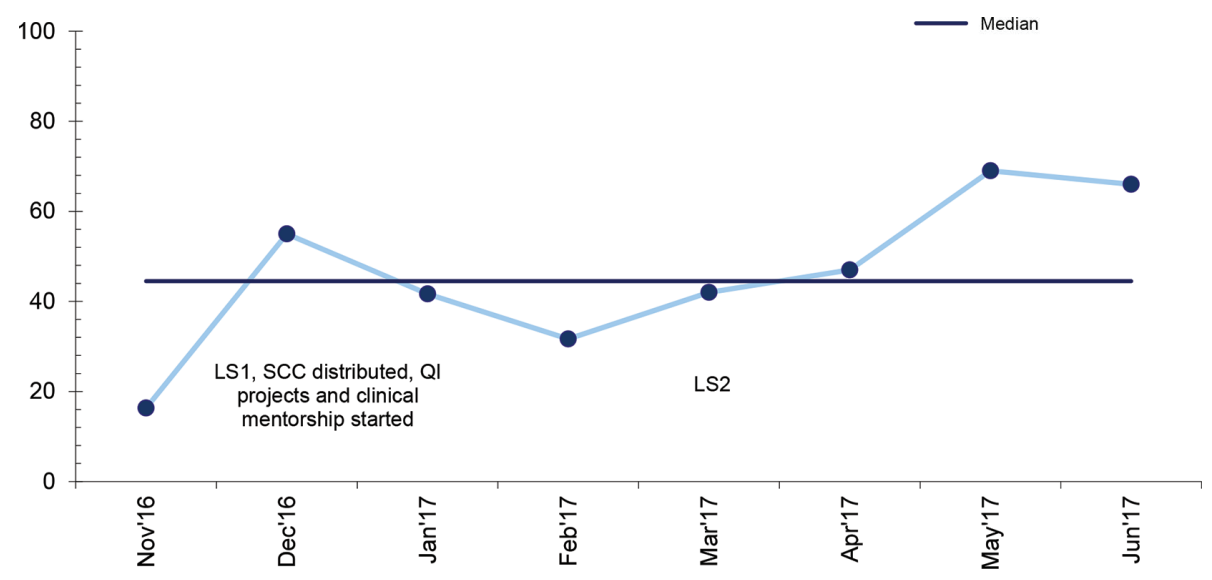

Abstract 865 Figure $6 \quad 100 \%$ completion of 'before pushing' bundle

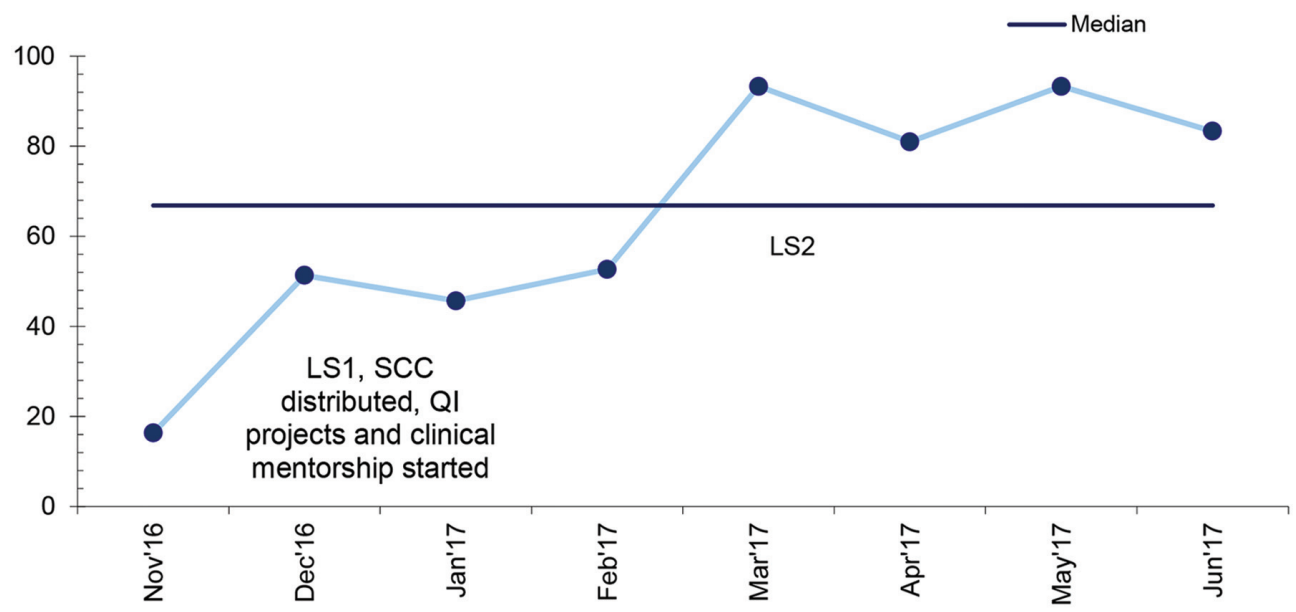

Abstract 865 Figure $7 \quad 100 \%$ completion of 'soon after birth' bundle

Conclusions Initial results are promising regarding program delivery and indicate high levels of facility-level QI activities, as well as initial signals of improved data and service quality in key maternal newborn health services.

\section{USING SIX SIGMA TO REDUCE READMISSIONS IN ELECTIVE TOTAL HIP \& KNEE ARTHROPLASTY}

${ }^{1}$ Brandon Hill, ${ }^{1}$ Eva Pittman, ${ }^{1}$ Hannah Lanier, ${ }^{1}$ Karen Hines, ${ }^{1}$ Charmaine Lewis, ${ }^{2}$ Jack Bowling, ${ }^{1}$ Caitlin Dunn, ${ }^{1}$ RoseAnn Gosswein, ${ }^{1}$ Shirley Glockner, ${ }^{1}$ David Oehler, ${ }^{1}$ Pam Cumber, 'Alice Matthews. ${ }^{1}$ New Hanover Regional Medical Centre, US; ${ }^{2}$ Bowling Orthopaedics, US

\subsection{6/bmjoq-2017-IHI.5}

Background New Hanover Regional Medical Centre is a public, not for profit, teaching hospital that performs over 2000 total joint arthroplasties per year. Following a near 1 million dollar penalty for excess 30 day Hip and Knee readmissions on the FY'15 CMS readmission report, a team was assembled to improve outcomes.

Objectives The team's objective was to identify trends among readmitted patients, conduct root cause analysis to determine process defects, identify risk factors within the population, optimise patients preoperatively, institute a risk scoring system to focus more energy and time with riskier patients, and decrease clinical variation that could lead to complications.

Methods Patient encounter data was used to conduct statistical analysis of medical diagnoses and demographic information to create a preoperative risk tool used to screen patients in preadmission testing. Risky patients were optimised, and received

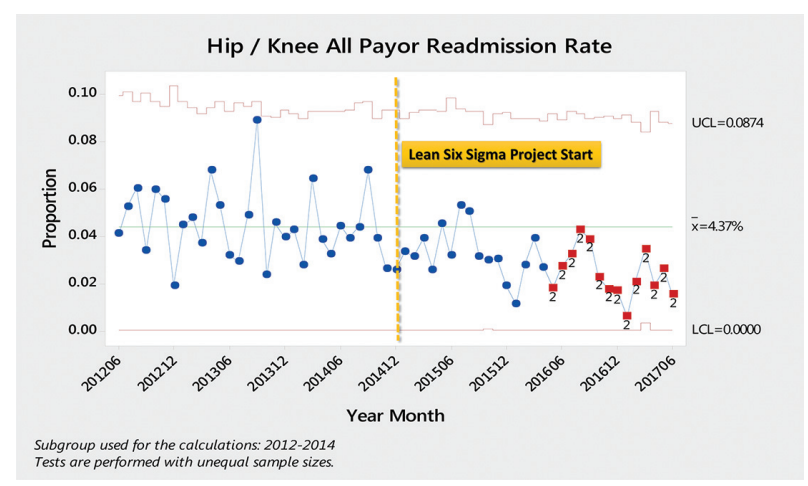

Abstract 869 Figure 1 Hip/knee all payor readmission rate 